\title{
CINCO CLAVES PRELIMINARES PARA EL ESTUDIO DEL PSICOANÁLISIS (LACANIANO) EN LA ESPAÑA DEL SEGUNDO FRANQUISMO Y LA TRANSICIÓN
}

\author{
Rodrigo Bilbao \\ Universidad de los Lagos, Académico Departamento de Ciencias Sociales. Chile \\ Email: rbilbaor@yahoo.com \\ ORCID iD: http://orcid.org/0000-0002-0795-235X \\ Rafael Huertas \\ Instituto de Historia - CSIC, Madrid, España \\ Email: rafael.huertas@cchs.csic.es \\ ORCID iD: http://orcid.org/0000-0002-4543-7180
}

Recibido: 1 noviembre 2018; Aceptado: 6 abril 2019.

Cómo citar este artículo/Citation: Bilbao, Rodrigo / Huertas, Rafael (2019), “Cinco claves preliminares para el estudio del psicoanálisis (lacaniano) en la España del segundo franquismo y la transición", Asclepio, 71(2): p281. https://doi.org/10.3989/asclepio.2019.22

RESUMEN: El presente artículo identifica y propone cinco claves a tener en cuenta para analizar la recepción y desarrollo del psicoanálisis en la España del segundo franquismo y la Transición: 1) la ausencia -o muy escasa presencia- de referencias psicoanalíticas en la producción psiquiátrica de los años cincuenta y sesenta; 2) las conexiones entre psicoanálisis, antipsiquiatría y estructuralismo; 3) la importancia de la llegada a España de Oscar Masotta, y su psicoanálisis "laico", en la consolidación de un discurso y una práctica psicoanalítica al margen de la IPA; 4) el peso de los psicoanalistas argentinos llegados al estado español tras el golpe de estado en su país; y 5) la importancia de la Revista de la Asociación Española de Neuropsiquiatría en difusión y trasmisión del psicoanálisis. Estas cinco claves agrupan situaciones y procesos que han sido estudiados con desigual profundidad, pero cuya consideración en conjunto parece imprescindible para obtener una visión global del proceso. Se concluye que las relaciones psiquiatría-psicoanálisis, la circulación del conocimiento, el contexto socio-político y cultural, etc., explican, entre otras cosas, las características de la recepción y desarrollo de un psicoanálisis post-freudiano en el que la orientación lacaniana tuvo preponderancia sobre otros enfoques o escuelas.

PALABRAS CLAVE: historia del psicoanálisis; Oscar Masotta; Jacques Lacan; freudomarxismo; Revista de la Asociación Española de Neuropsiquiatría; España; Transición democrática.

\section{FIVE PRELIMINARY KEYS TO THE STUDY OF PSYCHOANALYSIS IN SPAIN UNDER THE LATER FRANCO REGIME AND THE TRANSITION}

ABSTRACT: This article identifies and proposes five key elements to be taken into account in analysing the reception and development of psychoanalysis in Spain under the later Franco regime and during the Transition: 1) the absence - or very limited presence - of psychoanalytic points of reference in psychiatric production during the 1950s and 1960s; 2) the connections between psychoanalysis, anti-psychiatry and structuralism; 3) the importance of the arrival in Spain of Oscar Masotta, and his 'lay' psychoanalysis, in consolidating a psychoanalytic discourse and practice outside the IPA; 4) the influence of Argentinian psychoanalysts who came to Spain following the coup d'état in their country; and 5) the importance of the Revista de la Asociación Española de Neuropsiquiatría in disseminating and communicating psychoanalysis. These five keys group together situations and processes which have been studied to varying extents, but which it seems essential to consider as a whole in order to obtain an overall view of the process. We conclude that relations between psychiatry and psychoanalysis, the circulation of knowledge, and the sociopolitical and cultural context, etc. explain, among other things, the characteristics of the reception and development of a postFreudian psychoanalysis in which the Lacanian position predominated over other approaches and schools.

KEY WORDS: History of Psychoanalysis; Oscar Masotta; Jacques Lacan; Freudomarxism; Revista de la Asociación Española de Neuropsiquiatría; Spain; Democratic Transition.

Copyright: @ 2019 CSIC. Este es un artículo de acceso abierto distribuido bajo los términos de la licencia de uso y distribución Creative Commons Reconocimiento 4.0 Internacional (CC BY 4.0). 


\section{INTRODUCCIÓN}

En 1984, José Gutiérrez Terrazas publicaba en la Revista de la Asociación Española de Neuropsiquiatría un artículo titulado "Apuntes para un estudio sobre la historia del psicoanálisis en España" en el que abogaba por la necesidad de estudiar la recepción del psicoanálisis en España mediante trabajos históricos y reflexiones conceptuales que permitieran dar cuenta de la acogida y el desarrollo específicos que el descubrimiento del psicoanálisis ha tenido en nuestro país (Gutiérrez Terrazas, 1984). En la actualidad, a casi 35 años de aquella llamada de atención, el estado de la cuestión en torno al psicoanálisis es muy diferente; a los muy meritorios trabajos de Carles et al. (2000) o de Druet (2006) sobre la recepción institucional del psicoanálisis hay que añadir otras aportaciones recientes que marcan el acento en los usos del psicoanálisis en ámbitos diversos (criminología, sexualidad, etc.) y que están demostrando que su presencia -la del psicoanálisis- en España no fue tan limitada como se había supuesto (Lévy, 2016a; 2016b; Lévy y Huertas, 2018). En todo caso, siguen existiendo lagunas historiográficas importantes y una bibliografía relativamente escasa si la comparamos con otros aspectos de la historia de la psiquiatría y la psicología en España (Lázaro y Bujosa, 2000). Unas carencias que se hacen aún más notorias si nos preguntamos sobre el papel desempeñado por el psicoanálisis lacaniano en el cambio de la cultura psi que tuvo lugar durante el franquismo y la Transición; momentos de transformaciones sociales importantes, pero también de muy significativas novedades en la relación entre psiquiatría y psicoanálisis y en la articulación de sus encuentros y desencuentros. Nuestra intención en las páginas que siguen es valorar, y proponer, algunos de los elementos, o de las "claves", que habría que tener en cuenta para un estudio de la recepción del psicoanálisis en la época mencionada.

Si bien es cierto que, en general, la figura y la obra de Sigmund Freud ha sido la referencia fundamental para abordar la recepción y el desarrollo del psicoanálisis en las primeras décadas del siglo XX, la aparición de un pensamiento post-freudiano cada vez más rico y novedoso, viene a modular, de manera determinante, este tipo de estudios. La figura de Jacques Lacan aparece como un nuevo referente ineludible, aunque no único, en el impulso que el psicoanálisis recibe en la segunda mitad de la centuria. Para el caso español, la última etapa de la dictadura franquista y los años de la llamada Transición democrática configuran un escenario en el que nos parece posible identificar al menos cinco claves preliminares, cinco posibles caminos sobre los que transitar para analizar la presencia del psicoanálisis y sus peculiaridades concretas en un país sometido a un importante proceso de cambio político, social y cultural. Estas cinco claves podrían quedar sistematizadas del siguiente modo.

\section{SILENCIOS Y AUSENCIAS}

Según relata Anna-Cécile Druet (2014), sin duda la autora que mejor ha estudiado hasta el momento la recepción de Lacan en España, fue en el Instituto Pere Mata de Reus y de la mano de Francesc Tosquelles donde se leyó y comentó por primera vez en el estado español la obra del psicoanalista francés y, concretamente, su tesis doctoral De la psychose paranoïaque dans ses rapports avec la personnalité, en la que como se sabe se describe una paranoia de autocastigo a propósito del caso Aimée. Llama la atención que el curso que Tosquelles organizó en el Pere Mata sobre los contenidos de la tesis de Lacan se celebró en 1932, el mismo año de su presentación en París, lo que denota la actualización de los psiquiatras catalanes, a quienes les llegó el texto a través del historiador y médico de Reus Salvador Vilaseca (Tosquelles, 1975: 98). Sin embargo, tras este episodio puntual, durante los años siguientes la obra de Lacan aparece ausente en la literatura psiquiátrica española al menos hasta 1960. En los años cincuenta aparecen muy breves alusiones a este autor, como las de Henri Ey, el carismático psiquiatra francés que intentó, a través de un enfoque que denominó organodinamismo (Prats, 2001) un peculiar acercamiento entre la psiquiatría y el psicoanálisis (Garrabé, 1997). Ey publicó en las Actas Luso-española de Neurología y Psiquiatría, la revista fundada y dirigida por López Ibor, un artículo a la vez histórico y teórico, sobre la psiquiatría francesa durante la primera mitad del siglo $\mathrm{XX}$, en el que se alude a Lacan como una figura relevante del psicoanálisis francés (Ey, 1950); también en la revista que dirigía Ramón Sarró en Barcelona, en un artículo que refleja en buena medida la influencia de un pensamiento francés más amplio -las reminiscencias de la obra de Canguilhem por ejemplo-, se cita brevemente a Lacan como una figura destacada del panorama psiquiátrico y psicoanalítico francés (Ey, 1954). El propio López-Ibor, autor de La agonía del psicoanálisis (1951), llegó a publicar una reseña de 
un libro de Hécaen y Ajuriaguerra (1952) en la se refería a la imagen especular del niño -al concepto lacaniano del estadio del espejo- pero, tal como apunta con agudeza Anne-Cécile Druet (2014: 2), LópezIbor se limita a reproducir casi textualmente los contenidos del libro reseñado (López-lbor, 1953: 361), lo que le lleva a aventurar que los comentarios sobre Lacan del reputado psiquiatra español no eran consecuencia de una lectura directa de su obra.

A finales de la década de los cincuenta Lacan asistió al IV Congreso Internacional de Psicoterapia, celebrado en Barcelona, y en el que pronunció una conferencia titulada "La psychanalyse vrai et la fausse". Dicho congreso, organizado por Ramón Sarró, tuvo lugar entre el 1 y el 7 de septiembre de 1958 y es el primer encuentro internacional de psiquiatría celebrado en el estado español después de la guerra civil. Si en 1950, en el I Congreso Mundial de Psiquiatría, los psiquiatras que permanecieron en el país y los del exilio se habían encontrado por primera vez en un foro científico tras la contienda (Jordá, Rey, Angosto, 2007), ahora son las primeras figuras de la medicina mental europea las que acuden a Barcelona: Minkowski, Ey, Binswanger, y un largo etcétera de especialistas entre los que se contaba Lacan. Es evidente que un evento de estas características legitimaba al Régimen, lo que explica la gran cobertura informativa en la prensa o en el NODO, pero también la cuota local e ideológica impuesta por los organizadores: conferencia inaugural de Sarró, sección de psicoterapia y religión (inexistente en los congresos anteriores de Leiden, Londres y Zúrich), así como los amplios espacios dedicados en la prensa a comentar las intervenciones de los ponentes españoles, de López Ibor por ejemplo, en detrimento de los extranjeros. En este contexto, la intervención de Lacan pasó bastante desapercibida, pero como las de otros especialistas. Su ponencia tampoco fue publicada en las actas del congreso que acabaron apareciendo en la Revista de Psiquiatría y Psicología Médica de Europa y América Latinas, dirigida por Sarró, y no en Acta Psychotherapeutica Psychosomatica et Orthopaedagogica, como las del congreso anterior. Se ha especulado sobre las razones de la ausencia del texto de Lacan en las actas del congreso: censura política -difícil de sostener pues se trata de un texto muy teórico-; censura de la IPA -asimismo improbable, pues Lacan fue expulsado de la IPA, junto a Daniel Lagache y Françoise Dolto algunos años más tarde, en 1963-; olvido o extravío del documento, pero lo cierto es que las ponencias y comunicaciones publicadas fueron aproximadamente la mitad que las presentadas (Druet, 2014). Sea por criterios de selección, por dificultades organizativas, o por cualquier otra razón, lo cierto es que Lacan no fue el único autor cuya ponencia está ausente en dichas actas.

Merece la pena señalar, asimismo, que Lacan todavía no era, en los años cincuenta, la figura indiscutible en la que se convertirá en la década siguiente. Recuérdese que sus Écrits aparecen en Francia en 1966 y que el Seminario 11, el que inicia la enseñanza propiamente lacaniana, es de 1964 . Por eso, no tiene por qué sorprendernos las muy escasas referencias a Lacan en la literatura psiquiátrica española anterior a 1960 . Es cierto que comenzaba a ser un autor conocido y respetado en Francia, donde la vitalidad del psicoanálisis no puede compararse con su más que discreta presencia en España. Salvo algunas notables excepciones localizadas en ámbitos que no son los estrictamente clínicos (Lévy, 2016b), la psiquiatría oficial española fue, en la época que nos ocupa, bastante displicente con el psicoanálisis. Tan solo Ramón Sarró, cuyo interés por el mismo había ido decayendo (Sarró, 1956), intentó construir una psiquiatría antropológico-existencial en la que cabría el "rescate" de algunos postulados psicoanalíticos (Sarró Maluquer, 2006). Como indica Silvia Lévy (2016b: 146), los autores más citados en España fueron precisamente los que se habían alejado del psicoanálisis freudiano más ortodoxo para fundar otras escuelas o corrientes como la psicología individual de Adler o la psicología profunda de Jung, de contenidos más filosóficos y existenciales que sintonizaban mejor con los postulados de la psiquiatría hegemónica. Una psiquiatría que tuvo en la escuela de Heidelberg uno de sus referentes fundamentales y en la que autores como Karl Jaspers o Kurt Schneider fueron muy útiles para establecer un marco teórico que permitía, de la mano del primero oponerse tanto al psicoanálisis como al marxismo, y gracias al segundo disponer de un acabado sistema conceptual psiquiátrico (González de Pablo, 1987; 2016). Un sistema que podía aceptar algunas reminiscencias freudianas en el campo de la medicina psicosomática de Rof Carballo (Martínez López, 2008) o en la clínica de la neurosis, pero que, en lo referente a la locura propiamente dicha -a las psicosis- jamás contemplará el cruce entre psiquiatría y psicoanálisis que podían representar autores como Melanie Klein o Wilfred Bion, totalmente ausentes de las publicaciones psiquiátricas españolas, o como, por supuesto, Jacques Lacan. 
Vemos, pues, como los silencios en torno a algunos autores, su ausencia -o su muy limitada presencia- en las bibliografías, aunque pueden responder a razones muy diversas, ofrecen claves interpretativas que nos permiten valorar escenarios complejos en los que lo que no se dice cobra su importancia frente a lo que se dice.

\section{ANTIPSIQUIATRÍA, ESTRUCTURALISMO, PSICOANÁLISIS}

A finales de los años sesenta tienen lugar, como es sabido, novedades sociales y culturales importantes en el panorama nacional e internacional. Aunque de manera todavía limitada, el interés por un nuevo psicoanálisis empieza a detectarse en determinados núcleos de producción intelectual. En una fecha tan emblemática como 1968, se publica el que probablemente es el primer trabajo en castellano que analiza la obra de Lacan. Se trata de "Estructuralismo y psiquiatría" y su autor es un joven Josep Lluís MartíTusquet (1968), por aquel entonces colaborador de Sarró. El interés de este último por el estructuralismo es de sobra conocido, lector de Lévi-Strauss, pero también de Foucault, Althuser, Barthes o Saussure, fue uno de los organizadores del seminario sobre estructuralismo que en ese mismo año de 1968 se celebró en el Instituto Francés de Barcelona, en colaboración con la cátedra de Psiquiatría de la Universidad de Barcelona. El aludido trabajo de Martí-Tusquet es, en realidad, el comentario de una de las sesiones de dicho seminario, pero su interés por su obra le llevó no solo a invitar y recibir, junto con Sarró, a Lacan en Barcelona en 1972 -con motivo de su conferencia en la sesión inaugural de la Asociación de Psiquiatría de la Academia de Ciencias Médicas (Druet, 2014)-, sino a integrar el análisis estructural del psicoanálisis lacaniano a su reflexión sobre las instituciones psiquiátricas (Martí-Tusquets, 1971). Resulta muy interesante la relación que este autor establece entre la tesis lacaniana del inconsciente estructurado como un lenguaje y su aplicación a las comunidades terapéuticas (Martí-Tusquets, 1972a; 1972b), lo que nos permite establecer un nexo entre una determinada lectura de Lacan y los planteamientos antipsiquiátricos y de crítica institucional de la época. Martí-Tusquet es, sin duda, un autor interesante cuyas aportaciones merecerían un estudio en profundidad; baste aquí recordar que además de ser un referente de la psiquiatría social en los años setenta (Martí-Tusquets y
Obiols, 1976), presentó una ponencia al IV Congreso Nacional de Psicología, celebrado en Barcelona en 1973, en cuyos apoyos bibliográficos figuran autores como Bateson, y la teoría del doble vínculo, Laing o Esterson (Frigola, Martí-Tusquets, Palliser y Tous, 1973). Pero, sin duda, el dato más interesante es que uno de los firmantes de dicha ponencia era Carlos Frigola. Frigola había sido primero discípulo de Sarró y había completado su formación psicoanalítica en la Tavistock Clinic, donde conoció a Laing y Esterson. En 1977 fundó en Creixell (Gerona) una comunidad terapéutica inspirada en la de Kingsley Hall de Londres, que se denominó Existentalia y cuyo funcionamiento -basado en terapias grupales- duró dos años. Su conocimiento y vinculación con las experiencias británicas, así como su propia iniciativa fue reflejada por la prensa contracultural del momento, concretamente en la revista Ajoblanco, la publicación de la época en la que más claramente puede seguirse las relaciones entre antipsiquiatría y contracultura (Irisarri, 2017). Pero quizá lo más relevante, en relación con el psicoanálisis, sea su vinculación con Eva Reich -hija de Wilhelm Reich- con quien se analizó y con cuyo apoyo puso en marcha la Fundación Wilhelm Reich que agrupó un centro de planificación familiar, por deseo expreso y condición previa impuesta por Eva Reich, y la mencionada Existentalia. La comunidad terapéutica tuvo, como acabamos de indicar, un recorrido muy corto pero la Fundación sigue funcionando en la actualidad. Carlos Frigola es, probablemente, uno de los principales seguidores y divulgadores de Reich en el estado español, no solo a través de su práctica psicoterapéutica en la que incorporó principios reichnianos, o su colaboración con la revista y la Fundación SEXPOL, sino también con elaboraciones teóricas en las que, años más tarde, expone y analiza en detalle las teorías de Reich (Frigola 1981, 2016).

Pero la obra de Reich, como puede suponerse, no pudo pasar desapercibida a los psiquiatras más críticos de los años setenta. Ramón García, una de las figuras más relevantes del movimiento de contestación psiquiátrica durante el tardofranquismo y la transición (Huertas, 2017), contribuyó a la difusión del freudomarxismo, editando textos de Wilhelm Reich y de Igor Caruso (García, 1975), en un momento en el que las relaciones entre psicoanálisis y marxismo suscitaban un enorme interés. Unas relaciones estudiadas también por otras figuras carismáticas y progresistas de la psiquiatría española, como Carlos Castilla del Pino (1971). Cabe señalar también, para terminar este apartado, que fue Ramón García el que 
propició la publicación en castellano tanto de La institución negada de Franco Basaglia (1972), como del primer texto de Lacan (1970) publicado en España. Una manera de comprobar que, al menos en parte y durante este periodo, hubo núcleos comunes de recepción de psicoanálisis y antipsiquiatría, sobre los que merecería la pena profundizar.

\section{EL EFECTO MASOTTA}

Una tercera clave fundamental para entender el desarrollo del psicoanálisis en la España de la Transición tiene nombre propio: Oscar Masotta. Aunque existe una amplia bibliografía sobre este autor (García, 1980; Correas, 1991; Izaguirre, 1999; Scholten, 2001), y su papel en una cierta consolidación del psicoanálisis en España ha sido apuntado con acierto (Druet, 2017), sería necesario un estudio en profundidad de la labor desarrollada por Masotta en España, así como su significación y alcance. Masotta, una figura singular pues no era médico, ni psicólogo (las dos profesiones aceptada por la IPA), llega a Europa con un reconocido prestigio forjado en Argentina. Maestro, ensayista, intelectual de izquierdas y, en general, hombre de la cultura, había desarrollado en su país de origen una extensa labor de difusión del psicoanálisis lacaniano en distintos ámbitos culturales. La obra de Masotta recorre la crítica literaria, la filosofía, la semiología, pero también el arte pop (Masotta, 1967a) o el happening (Masotta, 1967b), y el comic, como organizador de la Primera Bienal Mundial de la Historieta (Gandolfo, 2013) y como autor de una obra específica (Masotta, 1970), todo ello como antesala de lo que serán sus ulteriores desarrollos en la difusión del psicoanálisis. No cabe duda que estos antecedentes le permitieron desempeñar un papel fundamental como promotor de un fecundo diálogo entre el psicoanálisis y el mundo de la cultura.

Tras fundar en 1974 la Escuela Freudiana de Buenos Aires, viajó a Londres primero y a Barcelona más tarde donde terminó instalándose en 1976, el mismo año del golpe de Estado que dio lugar a la dictadura militar en Argentina y, consecuentemente, muy poco antes de que empezaran a llegar al estado español profesionales argentinos que huían del terrorismo de Estado implantado en su país.

En 1977 se funda de manera oficial la Biblioteca Freudiana de Barcelona en la que Masotta y sus co- laboradores ponen en funcionamiento un sistema de difusión y enseñanza del psicoanálisis muy similar al que había propuesto unos años antes en Argentina y que, según Germán García, estaba compuesto por "intelectuales excluidos de la universidad (Masotta es uno de ellos), psicólogos excluidos del Psicoanálisis y Médicos sin didáctico, sobre la base de una economía asentada en los grupos de estudio y/o la derivación surgida de los mismos" (García, 1983, p. 76). A partir de ese momento, Masotta viaja por todo el país dictando conferencias, estableciendo lazos con otros grupos (sobre todo en Galicia y Andalucía) y creando un tejido de instituciones, grupos y discípulos. Es evidente que el refuerzo que supuso la llegada del importante contingente de psicoanalistas argentinos exiliados, a los que nos referiremos en el siguiente apartado, contribuyó a que estas iniciativas adquiriesen solidez y proyección, y a una relativa consolidación de un discurso y unas prácticas psicoanalíticas al margen de la IPA, a la que se sumaron discípulos españoles.

A toda esta labor organizativa habría que añadir una producción teórica específica, con obras, editadas en Buenos Aires y en Barcelona, que difunden, analizan e interpretan la obra de Lacan (Masotta, 1977a; 1977b; 1980; 1982) y cuyo estudio pormenorizado permitiría valorar la posición de nuestro autor en la historia del pensamiento psicoanalítico.

Concluyamos afirmando que el "efecto Masotta", que aquí nos hemos limitado a apuntar, aparece como un elemento clave, como un vector que amplía horizontes en la escena analítica. A partir de aquí cabe preguntarse, ahora sí con propiedad, sobre el papel desempeñado por el psicoanálisis en la cultura psi de aquellos años en España, para lo cual, obviamente, hizo falta no solo la influencia de una figura carismática, sino todo un movimiento colectivo.

\section{LA IMPRONTA DEL EXILIO ARGENTINO}

La brutal represión que se desencadenó tras el golpe de Estado de 1976 en Argentina afectó también al sector de la salud mental (Stagnaro y Conti, 2017). La Federación Argentina de Psiquiatras (FAP) fue incluida en la lista oficial de organizaciones subversivas y también fueron perseguidos, asesinados y detenidos-desaparecidos otros profesionales de la Asociación de Psicólogos de Buenos Aires (APBA) y de la Federación de Psicólogos de la República Argentina 
(FREPA) (Carpintero y Vainer, 2005). La llegada de un número importante de psicoanalistas argentinos a España, en condición de exiliados, precisamente en un momento de cambio social y cultural tan significativo, ejerció una impronta trascendental durante la Transición democrática.

Aunque se desconoce el número de psicoanalistas argentinos que llegaron a España, algunos autores lo han llegado a considerar como una "avalancha" (Averbach y Teszkiewicz, 2001). Nuevamente es Anne-Cécile Druet la investigadora que con más solidez ha estudiado este fenómeno; según esta autora, a partir de la llegada de los psicoanalistas argentinos, cualquier grupo de estudio, asociación o iniciativa cultural relacionada con el psicoanálisis en Madrid o Barcelona era altamente probable que incluyera al menos un argentino (que, de hecho, también era probable que fuera su principal organizador). En cuanto a cualquier grupo o actividad relacionado más específicamente con el lacanismo, la probabilidad de que su líder o promotor fuera alguien que hablaba con acento de Buenos Aires era aún más alto (Druet, 2012: 244). La figura del psicoanalista argentino se convirtió en un tópico que fue asimilado en el imaginario colectivo de un país que se abría con avidez a las novedades culturales.

No es que en España existiera un desierto psicoanalítico que ahora venía a rellenarse. Son conocidos los esfuerzos que, desde los años cincuenta y sesenta, estaban realizando, entre otros, Jerónimo Molina Nuñez -discípulo de Ángel Garma- y Ramón Portillo en Madrid, o de Pere Bofill, Pere Folch y Julia Corominas, fundadores de la Sociedad Luso-Español de Psicoanálisis en 1959, que lograron el reconocimiento de la IPA (Carles et al., 2000). Sin embargo, la llegada de la "avalancha" de psicoanalistas argentinos introdujo novedades importantes al menos en tres niveles de análisis: en primer lugar, la introducción de la orientación teórica lacaniana; en segundo lugar, la difusión del psicoanálisis como objeto cultural de largo alcance, pues los analistas argentinos intentaron recrear en España los intercambios entre el psicoanálisis y la esfera sociocultural tal como habían hecho en su país. No lo consiguieron, al menos con el alcance que llegó a tener en Buenos Aires, pero no se pueden olvidar los ecos culturales del lacanismo en España, con la creación de publicaciones como la Revista de literatura o Diwan (Druet 2008); finalmente, en tercer lugar, como ya hemos comentado en un apartado anterior, existió un notable interés por el psicoanálisis por parte de profesionales críticos implicados o comprometidos en las luchas políticas del tardofranquismo. Sin embargo, este interés se centraba en el uso subversivo que las interpretaciones y las prácticas psicoanalíticas pudieran tener, por eso, cuando se acercaron a los grupos españoles integrantes de la IPA debieron chocar con la rígida estructura jerárquica de los guardianes de una ortodoxia -burguesa, paternalista y convencional- que, además, había sabido convivir, aunque de modo subalterno, con la dictadura franquista. No es de extrañar, pues, que el halo de exiliados políticos y la práctica de un psicoanálisis "laico" por parte de los profesionales argentinos, propiciara una aproximación a los mismos de al menos parte de las generaciones más jóvenes de psiquiatras y psicólogos.

En este marco, resulta imprescindible señalar la febril actividad de este colectivo de psicoanalistas latinoamericanos y las numerosas iniciativas institucionalizadoras que protagonizaron. En Barcelona, antiguos discípulos y colaboradores de Masotta se reencontraron con su maestro y consolidaron un grupo importante e influyente. Así, como ya se ha indicado, el propio Masotta fundó en 1977 la Biblioteca Freudiana de Barcelona y Germán García, continuador de la labor de Masotta desde su fallecimiento en 1979, puso en marcha la Escuela de la Biblioteca Freudiana de Barcelona dos años más tarde. En Madrid también existieron iniciativas similares, como el Ateneo Freudiano de Madrid, creado en 1983 por Miriam Chrorme y Gustavo Dessal, o el grupo Analytica fundado en Madrid en 1979 y coordinado por José León Slimobich.

Destaquemos, asimismo, algunas publicaciones específicas que vieron la luz a primeros de los ochenta, entre las que encontramos Panthema, editada conjuntamente en Madrid, Pamplona y Granada, que fue el órgano de expresión de Analytica, pero sobre todo, la revista Sinthoma, editada en Barcelona y Serie Psicoanalítica en Madrid, ambas con un recorrido efímero, tan solo se publicaron entre 1981 y 1983, pero no por ello dejan de ser dos productos genuinos del fenómeno que estamos considerando. La primera conservaba el espíritu del psicoanálisis de Masotta, sus directores fueron Germán García y Jorge Jinkis y pretendió ser una revista no solo teórica sino accesible a un público general, una revista cultural de orientación psicoanalítica (lacaniana) en la que se publicaban textos de Lacan o del propio Masotta, pero también artículos teóricos, comentarios de libros, etc. 
La mayor parte de los autores son argentinos, aunque psicoanalistas de Barcelona, como Miquel Bassols o Vicente Palomera, colaboraron activamente con este proyecto. Ambos, junto a otros colegas catalanes, pusieron en marcha en 1983 la revista Otium diagonal que, editada por la Escuela Psicoanalítica de Barcelona, puede considerarse ya una iniciativa española, como lo fue la Biblioteca Galega de Estudios Freudianos, creada por los psicoanalistas lacanianos José Rodríguez Eiras y Fe Lacruz (1983), el primero de los cuales ejerció sus primeros años como psiquiatra en Oxford, donde conoció a Masotta y favoreció sus asentamientos en España.

Por su parte, Serie Psicoanalítica, fundada y dirigida por Jorge Alemán y Sergio Larriera en Madrid, tuvo una orientación un poco diferente, en la medida que primó la reflexión filosófica y epistemológica y dio cabida en sus páginas a autores que no necesariamente formaban parte del ámbito estrictamente psicoanalítico, como los filósofos Víctor Gómez Pin, Javier Echevarría o Agustín García Calvo. En ocasiones se propiciaron debates entre filósofos y psicoanalistas, como el protagonizado por Gustavo Bueno y Gustavo Dessal (1983). Es interesante, asimismo, la alusión directa a la cultura española al publicar el célebre texto de Ortega y Gasset "Psicoanálisis, ciencia problemática", seguido de un amplio análisis titulado "Discurso Científico y Discurso Psicoanalítico" a cargo de Jorge Alemán (1981).

La nómina de revistas psicoanalíticas aparecidas en la década de los ochenta puede ampliarse a otras cuantas: Ornicar? (versión castellana de la publicación francesa); la Revista Catalana de Psicoanálisi y la Revista de Psicoanálisis de Madrid (la primera lanzada por un grupo español reconocido por la IPA); Escansión (editada por Paidós en Buenos Aires y Barcelona); L'acudit (publicada íntegramente en catalán), etc. Todas ellas aparecen en el estudio descriptivo realizado por Druet (2007), un detallado trabajo de compilación documental que facilitará, sin duda, futuras investigaciones que estudien, interpreten y comparen los distintos núcleos de producción psicoanalítica a través de sus órganos de expresión. Soportes de conocimiento que surgen, en un primer momento y como hemos visto, por iniciativa -en general- de los psicoanalistas argentinos exiliados.

Con todo, una característica de todas estas publicaciones fue, como hemos apuntado, su muy efímera vida, la mayoría se editaron durante un lapso de tiempo muy corto (dos o tres años), dando lugar a colec- ciones de tan solo tres o cuatro números. A pesar del escaso material generado su conjunto constituye una fuente muy valiosa para apreciar la senda que el discurso y las corporaciones psicoanalíticas iban tomando en la España de la Transición. Sin embargo, es en otra revista, fundada también en 1981 por profesionales de la salud mental españoles, donde con más claridad puede valorarse la presencia del psicoanálisis en un espacio de reflexión que supo ser un punto de encuentro de ideas y culturas psi: la Revista de la Asociación Española de Neuropsiquiatría.

\section{LA REVISTA DE LA ASOCIACIÓN ESPAÑOLA DE NEUROPSIQUIATRÍA: SABERES PARCIALES}

En el congreso de la Asociación Española de Neuropsiquiatría, celebrado en Sevilla en 1977, tuvo lugar lo que se dio en llamar "la toma de la AEN", entendiendo como tal la llegada a la Junta Directiva de una candidatura mayoritariamente de izquierdas con un programa que propiciaría los cambios estatutarios necesarios para renovar la Asociación y convertirla en interlocutora de los poderes públicos en aspectos asistenciales, legislativos, etc. (Lázaro, 2000: 474; González de Chávez, 2003; Huertas, 2017). En un primer momento, se puso en marcha la publicación de un Boletín, pero a partir de 1981, tras el congreso de 1980 en Madrid, con Manuel González de Chávez como presidente de la AEN y Manuel Desviat como vocal de publicaciones, comenzó a editarse la Revista de la Asociación Española de Neuropsiquiatría, dirigida por Desviat desde su fundación hasta 1987. Como órgano de una asociación profesional, el enfoque y la línea editorial de la revista ha ido cambiando -o se han ido matizando- dependiendo de las distintas juntas directivas, directores y consejos de redacción que se han ido sucediendo, si bien puede decirse que siempre ha sido una publicación abierta a los distintos grupos profesionales, escuelas y tendencias que conviven en la Asociación y que su contenido refleja lo que ha sido una de las señas de identidad de la AEN: compromiso con la sociedad frente a la falacia cientificista, independencia, interdisciplinariedad, la defensa de la sanidad pública y una enseñanza y una investigación ligadas a la práctica (Fernández Liria, 1999).

Desde sus comienzos, la nueva publicación se convirtió en un punto de encuentro entre la psiquiatría y el psicoanálisis. El primer editorial, aparecido en el 
número 0 de la revista deja claro el talante de Manuel Desviat y su consejo de redacción:

No hay un Saber. Hay saberes parciales, andamiajes conceptuales que nos permiten aproximarnos al conocimiento de las cosas. Más aún en Psiquiatría, en Psicología, en Psicoanálisis, en cuanto que lo psíquico nos introduce en categorías situadas fuera del horizonte de la conciencia, en otra lógica difícilmente asimilable. Este es nuestro primer presupuesto. De ahí partimos, ajenos a todo reduccionismo, sea cual sea su signo, su ideología. Una multiplicidad de determinaciones da cuenta del hecho psíquico, del sufrimiento psicológico y del entramado social que constituye en cada época las Instituciones de la Salud Mental (Editorial, 1981: 1).

Pues bien, el psicoanálisis, uno de esos saberes parciales, va a tener una presencia destacada en las páginas de la revista. Una revista en la que se aludirá a Lacan y a otros referentes del psicoanálisis con naturalidad, y en la que el interés por el psicoanálisis se alejará de los círculos específicos para iniciados, convirtiéndose en interlocutor de la psiquiatría y en elemento importante para pensar la psicopatología en el marco de una cultura $p s i$ que se iba ensanchando al menos en algunos ámbitos.

Resulta imposible aquí analizar con cierta profundidad las aportaciones de contenido psicoanalítico publicadas en la Revista de la $A E N$, que sin duda deberían ser objeto de una investigación específica y detallada, pero si nos fijamos tan solo en sus primeros números podemos identificar una tendencia clara. Ya en el aludido $n$ o 0 la presencia del psicoanálisis resulta muy significativa. Inmediatamente después del Editorial al que nos hemos referido, el filósofo Víctor Gómez Pin (1981) abre con una "Justificación del Edipo" a partir de los Tres ensayos sobre teoría sexual de Freud, artículo al que sucede una "Reflexión en torno a la diferencia de los sexos" de Francisco Pereña (1981a), que se apoya en Freud y Lacan fundamentalmente, un tema que retornará en un trabajo posterior sobre "Freud y la sexualidad femenina" (Pereña, 1981b). A estos trabajos más teóricos hay que añadir el diálogo sobre la supervisión y su sentido en la formación de los psicoanalistas (Redondo, 1981a) o el artículo sobre el síntoma psicosomático en la infancia, en el que Freud y Lacan, pero también Dolto, Winnicott, Mannoni, Lebovici o Ajuriaguerra forman parte de un aparato crítico de clara orientación dinámica (Pedreira y Menéndez, 1981).

Destaquemos también cómo en números ulteriores se insiste en mostrar que "lo psicoanalítico está creciendo en nuestro medio", así lo afirmaba Pilar de
Miguel (1981: 48) cuando denota el interés por "una formación que durante largo tiempo ha tenido escasa difusión en nuestro país". Lo hace entrevistando a Fanny Elma de Shult, psicoanalista argentina perteneciente al contingente desplazado en la década de los 70 que llevaba trabajando los últimos cinco años en Madrid y Barcelona, lo que confirma una vez más la importancia otorgada al psicoanálisis latinoamericano en los medios españoles. Menos clínico y más cultural puede considerarse el "Comentario psicoanalítico de una autobiografía infantil: Les Mots, de Jean Paul Sartre" a cargo de Salvador Mascarell (1981), propuesta que estaría en sintonía con los esfuerzos por ampliar el discurso analítico más allá de la esfera de la práctica psiquiátrica o psicopatológica.

Una sección interesante de la revista, que nos ofrece información sobre la difusión del psicoanálisis en este espacio, es la Crítica de libros. Aunque las obras reseñadas son diversas, para el caso que nos ocupa, cabe citar, por ejemplo, el comentario de Fernando Colina (1981a) a una edición argentina de las Memorias de Schreber, una obra que pasando los años sería objeto de estudio -en colaboración con José María Álvarez (Álvarez y Colina, 2012)- y de edición por el autor de esta reseña en una de las colecciones de la AEN (Schreber, 2003). Asimismo, en los primeros números de la revista se informa de una traducción castellana de La teoría como ficción de Maud Mannoni (Redondo, 1981b), o de la conferencia en la Sociedad Psicoanalítica de Viena dictada en 1936 por Otto Fenichel -la recuperación de un clásico- y aparecida en el libro titulado Problemas de la técnica psicoanalítica (Redondo, 1981c). Especial interés tiene, a nuestro juicio, la reseña de Francisco Pereña de las Cinco conferencias caraqueñas sobre Lacan de 1979, realizada por su discípulo (yerno y más tarde albacea), quien ya estaba en ese momento tomando el lugar de máximo representante del movimiento lacaniano, JacquesAlain Miller. Esta figura no es cualquiera en el lacanismo en general -por como continuó la historia hasta nuestros días-, ni en particular en España por cómo se desarrolló y consolidó la orientación lacaniana a la muerte de Masotta. Esto ya es otra historia, pero lo que se debe resaltar en este momento es que en esas Cinco Conferencias desarrolladas en Caracas (último destino Internacional de Lacan) MiIler expone los elementos fundamentales de la teoría de Lacan desde la epistemología y el lenguaje, hasta la transferencia (Pereña, 1981c). 
Esta última reseña viene a enlazar, en cierto modo, con un artículo del propio Pereña, publicado en el número siguiente de la Revista de la $A E N$, en el que con el título "Presentación de Lacan" realiza una hermosa declaración de fundamentos al decir que "Lacan merece ser leído sin profetas pues nadie posee su justa interpretación. El testimonio de Lacan no tiene herederos, no hay lacanianos" (Pereña, 1981d: 8). No es difícil imaginar en estas palabras una especie de respuesta -de resistencia- al monopolio milleriano, máxime cuando este autor demostrará, en su posterior trayectoria, un pensamiento independiente $y$ creativo que ha dado lugar a una amplia producción (Pereña, 2001; 2002; 2006).

La recepción de Lacan en las páginas de la Revista de la AEN tuvo también otras expresiones, como en el artículo de Fernando Colina "Del amor y otras psicosis", con un claro guiño al psicoanálisis lacaniano: "Si el Yo es el síntoma (Lacan), el amor es nuestra enfermedad, nuestra psicosis" (Colina, 1981b: 57). Al igual que Francisco Pereña, Fernando Colina, que asumió la dirección de la revista más tarde (entre 1994 y 1999), será otro de los baluartes del psicoanálisis en la AEN. Su obra posterior resulta igualmente de gran trascendencia (Colina, 2001, 2006; 2011; 2014). No deja de resultar esclarecedor, y por demás interesante, que dos de los colaboradores más activos de la Revista de la $A E N$, introductores del psicoanálisis en España a través de este soporte se convirtieran más tarde en intelectuales reconocidos y referentes importantes de una determinada manera de entender la clínica psicopatológica y las relaciones entre subjetividad y cultura.

No fueron los únicos, la contribución de María Redondo fue importante en los primeros años de la revista, así como la de Vicente Mira, que llegó a ser traductor de Lacan y Presidente de la Asociación de Psicoanálisis del Campo Lacaniano. De entre sus colaboraciones en la revista destacaremos sus "Reflexiones en torno a la violencia infantil", en las que además de Lacan, recurrirá a Klein y Winnicott (Mira, 1981).

Como se ve, y tan solo hemos revisado los números correspondientes al primer año, la Revista de la AEN constituye sin duda una fuente de primer orden para la historia del psicoanálisis en la España de la Transición. Una fuente que permite, además, valorar el papel que termina desempeñando el psicoanálisis en un marco teórico y profesional alternativo y reformista. Un texto clave en este sentido es el que firma el propio director de la revista sobre "Psiquiatría y po- lítica en España: La salud mental en el marco de la salud pública". Manuel Desviat analiza en este artículo la situación española -como antesala de la reforma que se iniciará en 1985-, reconociendo y reivindicando la ruptura provocada por Freud antes del "equívoco término de antipsiquiatría", toda vez que "la ruptura de esa precisa taxonomía que establecía los límites entre lo normal y anormal, está ya en Freud en la medida que demuestra que el paciente psíquico se inscribe en una psicología del mismo orden que el considerado sano" (Desviat, 1981: 113).

Una última reflexión, en espera de una investigación más detallada al respecto, nos la suscita un texto sobre "La formación de los médicos residentes en psiquiatría. Acerca de la enseñanza del psicoanálisis" (Leal, Pereña y Redondo, 1982). Se trata, en realidad de dos aportaciones publicadas en la sección de Debate; el primero, firmado por Fernando Leal, despliega el tema de la formación de psiquiatras, sus directrices y condiciones del momento; el segundo, de la mano de nuevamente de Francisco Pereña y María Redondo (colaboradores asiduos de los primeros números), discuten el lugar del psicoanálisis en la ciencia, o dicho de otro modo, analizan la enseñanza del psicoanálisis y su estatuto científico. En el índice de la revista aparecen juntos los títulos y los tres como autores, cuando claramente son dos textos independientes que aparecen como documentos para un debate que, finalmente, queda a cargo del lector. Como quiera que sea, lo interesante es lo que se desea trasmitir, aunque ese deseo solo se reconozca entre líneas, casi como un lapsus: ¿podrá existir un punto de encuentro entre psicoanálisis y psiquiatría en la formación misma de los psiquiatras?; ¿el psicoanálisis y su estatuto científico permiten un lugar en la psiquiatría o en la política de la salud mental española?, tal como se planteaba Desviat; o quizás, por último, ¿sería posible escribir (e inscribir) en un solo texto (y no en dos) la relación entre psiquiatría y psicoanálisis en España, o esto resulta imposible? Preguntas que quedan abiertas y que, en muy buena medida, siguen vigentes hoy día.

En las páginas precedentes hemos identificado cinco claves que nos parecen fundamentales para entender el desarrollo del psicoanálisis en la España del segundo franquismo y la Transición democrática. Se trata de cinco claves que agrupan situaciones y procesos que han sido estudiados con desigual profundidad -los trabajos de Anna-Cécile Druet han sido, 
sin duda, los más relevantes-, pero cuya consideración en conjunto parece imprescindible para obtener una visión global de unas dinámicas complejas en las que elementos muy diversos se complementan o se atraviesan con frecuencia.

Las relaciones psiquiatría-psicoanálisis, la circulación del conocimiento, el contexto socio-político y cultural, etc., explican, entre otras cosas, las características de la recepción y desarrollo de un psicoanálisis post-freudiano en el que la orientación lacaniana tuvo preponderancia sobre otros enfoques o escuelas. Convendrá, en todo caso, valorar la importancia e

\section{BIBLIOGRAFÍA}

Alemán, Jorge (1981), "Discurso científico y discurso psicoanalítico", Serie Psicoanalítica, 2-3, pp. 7-36.

Álvarez, José María; Colina, Fernando (2012), “Daniel Paul Schreber, profesor de psicosis", Átopos, 13, pp. 103-111.

Averbach, Marina; Teszkiewicz, Luis (2001), "Psicoanalistas argentinos en la salud mental española", Disponible en: http://www. monografias.com/trabajos911/capitulo-libropsiquiatria/capitulo-libro-psiquiatria.shtml. (consultado el 7 de junio de 2018)

Ávila, Alejandro (2015), "Psychoanalysis in Spain: A Brief Account of the Transformative Pathways batwean the Psychoanalysts and their institutions", Psychoanalytic Inquiry, 35, pp. 234-239.

Basaglia, Franco (1972), La institución negada. Informe de un hospital, Barral, Barcelona.

Carles, Francisco; Muñoz, Isabel; Llor, Carmen y Marset, Pedro (2000), Psicoanálisis en España (1893-1968), Madrid, Asociación Española de Neuropsiquiatría.

Carpintero, Enrique; Vainer, Alejandro (2005), Las huellas de la memoria. Psicoanálisis y salud mental en la Argentina en los '60 y '70, Buenos Aires, Topía.

Castilla del Pino, Carlos (1971), Psicoanálisis y marxismo, Alianza, Madrid.

Colina, Fernando (1981a), "Presentación y enseñanzas de Schreber", Revista de la Asociación Española de Neuropsiquiatría, 1 (0), pp. 134-136.

Colina, Fernando (1981b), "Del amor y otras psicosis", Revista de la Asociación Española de Neuropsiquiatría, 1 (2), pp. 57-76.

Colina, Fernando (2001), El saber delirante, Madrid, Síntesis.

Colina, Fernando (2006), Deseo sobre deseo, Madrid, Cuatro.

Colina, Fernando (2011), Melancolía y paranoia, Madrid, Síntesis.

Colina, Fernando (2014), Sobre la locura, Valladolid, Cuatro. influencia de esta orientación, que convivió con la supuesta ortodoxia de la IPA, pero también con otros acercamientos importantes como el freudomarxismo o como, algún tiempo más tarde, el psicoanálisis relacional (Ávila, 2015).

\section{AGRADECIMIENTOS}

Trabajo realizado en el marco de los Proyectos de Investigación HAR2015-66374-R (MINECO/FEDER) y RTI2018-098006-B-I00 (MICIU/FEDER)

Consejo de Redacción (1981), "Editorial”, Revista de la Asociación Española de Neuropsiquiatría, 1 (0), pp. 3-4.

Correas, Carlos (1991), Operación Masotta. Catálogos. Buenos Aires, Catálogos.

Dessal, Gustavo (1983), "Respuesta a Gustavo Bueno", Serie Psicoanalítica, 2-3, pp. 227-246.

Desviat, Manuel (1981), “Psiquitaría y política en España (La salud mental en el marco de la salud pública)", Revista de la Asociación Española de Neuropsiquiatría, 1 (2), pp. 110-120.

Druet, Anne-Cécile (2006), La psychanalise dans l'Espagne postfranquiste (1975-1985), Tesis doctoral, Paris, Université Paris IV-Sorbonne.

Druet, Anne-Cécile (2007), "Estudio descriptivo de las revistas de psicoanálisis en España hasta 1985", Archivos de Psiquiatría, 70 (2), pp. 115-130.

Druet, Anna-Cécile (2008), "Ecos socioculturales de la introducción del lacanismo en España", Cahiers de LI.RI.CO, 4, pp. $165-174$

Druet, Anne-Cécile (2012), "The transatlantic element: psychoanalysis, exile, circulation of ideas and institutionalitation between Spain and Argentina", Psychoanalysis and History 14(2), pp. 237-251.

Druet, Anne-Cécile (2014), “La psiquiatría española y Jaques Lacan antes de 1975", Asclepio, 66 (1), pp. 1-10.

Druet, Anne-Cécile (2017), "Psicoanálisis y transición democrática en España”, História, Ciencia, Saúde - Manguinhos, 24 (supl), pp. 63-78.

Eiras, José; Lacruz, Fe. (1983), “Biblioteca Galega de Estudios Freudianos", Analytica, 34, pp. 77-78.

Ey, Henri (1950), “La psiquiatría francesa de 1900 a 1950”, Actas Luso-españolas de Neurología y Psiquiatría, 9 (2), pp. 73-82.

Ey, Henri (1954), "Lo normal y lo patológico en psiquiatría", Revista de Psiquiatría y Psicología Médica de Europa y América Latinas, 1 (5), pp. 325-334. 
Fernández Liria, Alberto (1999): "La AEN y la Psiquiatría española a finales del siglo XX", Revista de la Asociación Española de Neuropsiquiatría, 19 (71), pp. 503-20.

Frigola, C., Martí-Tusquet, J.L., Palliser, P. y Tous, J.M. (1973), "Toma de decisiones en familias normales y anormales", Anuario de Psicología, 9, pp. 75-90.

Frigola, Carlos (1981), El autor y su obra. Wilhelm Reich, Barcelona, Barcanova.

Frigola, Carlos (2016), Cartas de Freud a Reich. El compromiso político y social del psicoanálisis, Barcelona, Laertes.

Gandolfo, Amadeo (2013). “Código y estilo: Oscar Masotta como teórico del comic. Del intelectual comprometido al estilista interior", Luthor, 13, febrero, Buenos Aires. Artículo en Línea: http://www.revistaluthor.com.ar/spip. php?article68 (consultado el 11 de julio de 2018)

García, Germán (1980), Oscar Masotta y el psicoanálisis en castellano, Barcelona, Argonauta.

García, Germán (1983), “Oscar Masotta y el análisis laico”, En Sinthoma, 2-3, pp. 69-89.

García, Ramón (1975). Psicoanálisis y sociedad. Apuntes de freudomarxismo. Anagrama, Barcelona.

Garrabé, Jean (1997), Henri Ey et la pensée psychiatrique contemporaine, Paris, Synthélabo.

Gómez Pin, Víctor (1981), “Justificación del Edipo”, Revista de la Asociación Española de Neuropsiquiatría, 1 (0), pp. 5-27.

González de Chávez, Manuel (2003), “La generación de la democracia: De la Coordinadora psiquiátrica a la Asociación Española de Neuropsiquiatría", Frenia, 3 (1), pp. 87-114.

González de Pablo, Ángel (1987), Sobre las génesis del orden psiquiátrico en la posguerra española: la implantación de la escuela de Heidelberg en España", Revista de la Asociación Española de Neuropsiquiatría, 7 (23), pp. 633-647.

González de Pablo, Ángel (2016), “La teoría psiquiátrica durante el primer franquismo", en Campos, R. y González de Pablo, A. (coords.), Psiquiatría e higiene mental en el primer franquismo, Madrid, los libros de la Catarata, pp. 46-80.

Gutiérrez Terrazas, José (1984), "Apuntes para un estudio sobre la historia del psicoanálisis en España", Revista de la Asociación Española de Neuropsiquiatría, 4 (10), pp. 207-221.

Hécaen, Henri; Ajuriaguerra, Julián (1952), Méconnaissances et hallucinations corporelles. Intégration et désintégration de la somatognosie, Paris, Masson.

Huertas, Rafael (2017), "Psiquiatría, antipsiquiatria y movilización social". En: Huertas, R. (coord.) Psiquiatría y antipsiquiatría en el segundo franquismo y la Transición, Madrid, Los libros de la Catarata, pp. 80-104.

Irisarri, Fabiola (2017), "Contracultura y antipsiquiatría: el caso de Ajoblanco". En: Huertas, R. (coord.) Psiquiatría y antipsiquiatría en el segundo franquismo y la Transición, Madrid, Los libros de la Catarata, pp. 162-191.

Izaguirre, Marcelo (1999), Oscar Masotta. El revés de la trama, Buenos Aires, Editorial Atuel / Anáfora.
Jordà Moscardó, Enrique; Rey, Antonio y Angosto Saura, Tiburcio (2007), "La psiquiatría franquista y del exilio en el I Congreso Mundial de Psiquiatría. París". En: Campos, Ricardo; Villasante, Olga; Huertas, Rafael, (eds.), De la edad de Plata al exilio. Construcción y reconstrucción de la psiquiatría española, Madrid, Frenia, pp. 269-285.

Lacan, Jacques (1970), El objeto del psicoanálisis. Barcelona, Anagrama.

Lázaro, José (2000), “Historia de la Asociación Española de Neuropsiquiatría (1924-1999)", Revista de la Asociación Española de Neuropsiquiatría, 20, pp. 397-515.

Lázaro, José; Bujosa, Francesc (2000), Historiografía de la psiquiatría española, Madrid, Triacastela.

Leal, Fernando; Pereña, Francisco y Redondo, María (1982), “"La formación de los médicos residentes en psiquiatría. Acerca de la enseñanza del psicoanálisis", Revista de la Asociación Española de Neuropsiquiatría, 2 (5), pp. 75-95.

Lévy, Silvia (2016a), "Delitos inconscientes. Psicoanálisis y teoría penal durante la Segunda República española", Culturas Psi/Psy Cultures, 6, pp. 34-64.

Lévy, Silvia (2016b), “Prevenir, rehabilitar y sancionar. La incorporación de las ideas psicoanalíticas a la psiquiatría forense (1930-1950), en Campos, R. y González de Pablo, A. (coords.), Psiquiatría e higiene mental en el primer franquismo, Madrid, los libros de la Catarata, pp. 145-174.

Lévy, Silvia; Huertas, Rafael (2018), "From outrage to normalization: Uses of psychoanalysis by the Spanish sexual reform movement (1920-1939)", Cuadernos de Historia Contemporánea, 40, pp. 33-49.

López Ibor, Juan José (1951), La agonía del psicoanálisis, Madrid, Espasa-Calpe.

López Ibor, Juan José (1953), “Méconnaissances et hallucinations corporelles [reseña]", Actas Luso-españolas de Neurología y Psiquiatría, 12 (4), p. 361.

Martí-Tusquets, Josep Lluís (1968), “Estructuralismo y psiquiatría", Revista de Psiquiatría y Psicología Médica de Europa y América Latinas, 8 (5), pp. 235-236.

Martí-Tusquets, Josep Lluís (1971), "Influencia del análisis estructural en la evolución terapéutica del hospital psiquiátrico", Revista de Psiquiatría y Psicología Médica de Europa y América Latinas, 10 (4), pp. 208-209.

Martí-Tusquets, Josep Lluís (1972a), “Estructura de los grupos terapéuticos", Salud Mental, 5 (9), pp. 4-9.

Martí-Tusquets, Josep Lluís (1972b), “El equipo terapéutico y el trabajo en grupo", Salud Mental, 5 (9), pp. 19-25.

Martí-Tusquets, J.L.; Obiols, J. (1976), Psiquiatría social, Barcelona, Herder.

Martínez López, Francisco (2008), Juan Rof Carballo y la medicina psicosomática, Madrid, Díaz de Santos.

Mascarell, Salvador (1981), “Comentario psicoanalítico de una autobiografía infantil: Les Mots, de Jean Paul Sartre", Revista de la Asociación Española de Neuropsiquiatría, 1 (1), pp. 4-15. 
Masotta. Oscar (1967a), El pop art, Buenos Aires, Columba.

Masotta, Oscar (1967b), Happening, Buenos Aires, Ed. Jorge Álvarez.

Masotta, Oscar (1970), La historieta en el mundo moderno, Buenos Aires, Paidos.

Masotta, Oscar (1977a), Introducción a la lectura de Jacques Lacan, Buenos Aires, Proteo.

Masotta, Oscar (1977b), Ensayos lacanianos, Barcelona, Anagrama.

Masotta, Oscar (1980), El modelo pulsional, Buenos Aires, Altazor.

Masotta, Oscar (1982), Lecciones de psicoanálisis. Colección Extensión Freudiana, Barcelona, Argonauta.

Miguel, Pilar de (1981), “Acerca de la formación psicoanalítica. Entrevista a Fanny Elman de Schutt", Revista de la Asociación Española de Neuropsiquiatría, 1 (1), pp. 48-55.

Mira, Vicente (1981), "Reflexiones en torno a la violencia infantil", Revista de la Asociación Española de Neuropsiquiatría, 1 (2), pp. 77-88.

Pedreira, José Luis; Menéndez, Federico (1981), "El síntoma en la infancia: valoración psicodinámica", Revista de la Asociación Española de Neuropsiquiatría, 1 (0), pp. 81-95.

Pereña, Francisco (1981a), "Reflexiones en torno a la diferencia de sexos", Revista de la Asociación Española de Neuropsiquiatría, 1 (0), pp. 28-38.

Pereña, Francisco (1981b), "Freud y la sexualidad femenina", Revista de la Asociación Española de Neuropsiquiatría, 1 (2), pp. 9-30.

Pereña, Francisco (1981c), "Cinco conferencias caraqueñas sobre Lacan", Revista de la Asociación Española de Neuropsiquiatría, 1 (1), pp. 119-120.

Pereña, Francisco (1981d), "Presentación de Lacan”, Revista de la Asociación Española de Neuropsiquiatría, 1 (2), pp. 4-8.

Pereña, Francisco (2001), La pulsión y la culpa, Madrid, Síntesis.
Pereña, Francisco (2002), El hombre sin argumento. Una introducción a la clínica psicoanalítica, Madrid, Síntesis.

Pereña, Francisco (2006), Soledad, pertenencia y transferencia, Madrid, Síntesis.

Prats, Philippe (2001), Une psychiatrie philosophique: l'organodynamisme comme anthropologie, Paris, L'Harmattan.

Redondo, María (1981a), “Acerca de la supervisión: entrevista con Carlos Sopena", Revista de la Asociación Española de Neuropsiquiatría, 1 (0), pp. 73-80.

Redondo, María (1981b), "La teoría como ficción”, Revista de la Asociación Española de Neuropsiquiatría, pp. 137-138.

Redondo, María (1981c), "Problemas de la técnica psicoanalítica", Revista de la Asociación Española de Neuropsiquiatría, 1 (1), pp. 117-118.

Sarró Maluquer, Ramón (2006), “Las formas elementales de la vida esquizofrénica: las dos antropologías en el pensamiento de Ramón Sarró Burbano", Frenia, 6, pp. 109-126.

Sarró, Ramón (1956), “El ocaso del psicoanálisis ortodoxo en Sudamérica", Revista de Psiquiatría y Psicología Médica de Europa y América Latina 2 (7), pp. 631-636.

Scholten, Hernán (2001), Oscar Masotta y la fenomenología. Un problema en la historia del psicoanálisis. Buenos Aires, Atuel-Anáfora.

Schreber, Daniel Paul (2003), Sucesos memorables de un enfermo de los nervios, Madrid, Asociación Española de Neuropsiquiatría.

Stagnaro, Juan Carlos; Conti, Norberto (2017), "La salud mental en Argentina en los años del plomo (1976-1983)" En Huertas, Rafael (coord.), Políticas de salud mental y cambio social en América Latina, Madrid, Los libros de la Catarata, pp. 23-55.

Tosquelles, Francesc (1975), “À propos de...la réédition de la thèse de Jacques Lacan", Psychiatries, 21, pp. 93-98. 